\title{
Review of Meniscal Allograft Transplantation Focusing on Long-term Results and Evaluation Methods
}

\author{
Bum-Sik Lee, $\mathrm{MD}^{1}$, Jong-Min Kim, $\mathrm{MD}^{2}$, Dong-Wook Sohn, $\mathrm{MD}^{2}$, and Seong-Il Bin, $\mathrm{MD}^{2}$ \\ ${ }^{1}$ Department of Orthopedic Surgery, Incheon St. Mary’s Hospital, The Catholic University of Korea College of Medicine, Incheon; ${ }^{2}$ Department of Orthopedic Surgery, \\ Asan Medical Center, University of Ulsan College of Medicine, Seoul, Korea
}

\begin{abstract}
With recognition of the biomechanical role of the meniscus, such as load distribution and joint stability in the knee joint, there has been a shift in the treatment of meniscal tears from open total meniscectomy to preservation of the meniscal functions as much as possible with symptomatic relief. Recently, technical development of meniscal surgery, with advanced arthroscopic equipment and instruments, enables biological reconstruction of load bearing functions in the meniscus deficient knee through allograft tissue transplantation as well as repair of torn menisci. Meniscal allograft transplantation (MAT) has been considered as one of the few viable treatment options for the young meniscectomized knees based on various animal experiments and clinical studies. Still, there is insufficient evidence for the long-term chondroprotective effect of human MAT. Some longterm follow-up studies showed that the technique resulted in graft degeneration, deformation, and tear, and structural changes in the remodeling process in early MAT cases, disrupting functional restoration of the original meniscus. Nevertheless, advanced outcomes are documented in some recent studies. The purpose of this article is to review the mid- and long-term follow-up results of MAT and to improve understanding of MAT with evaluation methods of meniscal transplants using magnetic resonance imaging or second-look arthroscopy.
\end{abstract}

Keywords: Knee, Menisci, Transplantation

\section{Introduction}

The goal of the treatment of torn menisci was symptomatic relief through open total meniscectomy in the past. However, the surgical treatment has been shifted for preservation of meniscal functions as well as symptomatic improvement ${ }^{1,2)}$ with growing recognition of the importance of biomechanical functions of the meniscus, such as the chondroprotective effect $^{3,4)}$ through load distribution and knee joint stabilization ${ }^{5-7)}$. Currently, transplantation of allograft meniscal tissue is used for reconstruction of biomechanical properties of the meniscus

Received January 16, 2013; Accepted February 1, 2013

Correspondence to: Seong-Il Bin, MD

Department of Orthopedic Surgery, Asan Medical Center, University of Ulsan College of Medicine, 88 Olympic-ro 43-gil, Songpa-gu, Seoul 138736, Korea

Tel: +82-2-3010-3528, Fax: +82-2-488-7877

E-mail: sibin@amc.seoul.kr

This is an Open Access article distributed under the terms of the Creative Commons Attribution Non-Commercial License (http://creativecommons.org/licenses/by-nc/3.0/) which permits unrestricted non-commercial use, distribution, and reproduction in any medium, provided the original work is properly cited. in the meniscus-deficient knee with the aid of advanced arthroscopic equipment, instruments, and techniques ${ }^{8-11)}$.

The ideal long-term result of meniscal allograft transplantation (MAT) is delay of articular cartilage degeneration, which is still to be proven by substantial evidence ${ }^{12,13)}$. Although some animal model studies have shown that MAT can restore chondroprotective function of the meniscus to a certain level ${ }^{14,15)}$, the transplanted menisci failed to provide complete restoration of the functions of the original meniscus. In most studies of the early MAT cases, significant degeneration, deformation, and tear, and structural changes of the transplant were observed during the remodeling process ${ }^{16-18)}$. However, MAT is considered as one of the effective alternative options for symptomatic meniscectomized knees because there are few viable treatment strategies in these patients.

Although there are a number of research and literature on MAT, it is not easy to assess their outcomes due to the small number of subjects, various meniscus preservation techniques, operative methods, and indications, various concomitant procedures including anterior cruciate ligament (ACL) reconstruction, high tibial osteotomy (HTO), and cartilage repairing procedures, in addition to the absence of established evaluation methods. The 


\section{Lee et al. Review of Meniscal Allograft Transplantation Focusing on Long-term Results and Evaluation Methods}

purpose of this article is to improve the understanding of MAT with a review of the mid- and long-term follow-up results and recent published data using objective evaluation methods of meniscal transplants.

\section{Long-term Follow-up Results of Early MAT Cases}

The established purpose of human MAT is to relieve pain and improve functions in the meniscectomized $\mathrm{knee}^{8-10,12,13)}$. In addition, the orthopedic surgeons now expect the meniscal transplants to provide a chondroprotective effect for joint preservation in the long-term follow-up. In 1984, Milachowski et al. ${ }^{19)}$ first performed MAT in ACL reconstruction on human subjects in an attempt to promote synergistic effects by restoring secondary restraint function of the posterior horn of the medial meniscus against anterior instability based on their animal experiments in sheep. In their study, only three transplanted menisci had to be removed out of 22 cases for a minimum 14 months of follow-up. Wirth et al. ${ }^{16)}$ evaluated the clinical outcome of MAT in 23 cases consisting of 22 cases in the abovementioned study and 1 additional case with a lyophilized graft at 3 years and 14 years postoperatively. The overall results were satisfactory although the Lysholm score decreased from 84 points at 3 years postoperatively to 75 points at 14 years postoperatively. They compared the 23 MATs with two control groups that underwent isolated ACL reconstruction without MAT (one with previous experience of meniscectomy and the other one with intact menisci). The clinical results were better in the deep frozen graft group than the lyophilized graft group. The lyophilized graft exhibited more shrinkage than deep frozen grafts on magnetic resonance imaging (MRI) and second-look arthroscopy. The results of the lyophilized graft group were more comparable to the meniscus-deficient control group, whereas the deep frozen graft group produced more similar results to the control group with intact meniscus. Of these Milachowski's cases, 5 cases with a deep frozen graft were evaluated at 20 years postoperatively by von Lewinski et al. ${ }^{17}$. The Lysholm score ranged from 21 to 97 points, indicating the clinical results were good in some cases. Although the overall results were satisfactory, clear radiographic evidence of degeneration was observed in all patients: the Kellgren-Lawrence grade was 2 in 2 patients, 3 in 2 patients, and 4 in only 1 patient.

In another recent report by Binnet et al. ${ }^{18)}$, all of the 4 patients had grade 4 degenerative arthritis at 19 years after lyophilized graft transplantation combined with revision ACL reconstruction, indicating the importance of graft preservation. Currently, lyophilized grafts are rarely used in meniscus transplantation due to the risk of significant changes in the tissue properties and severe shrinkage of meniscal transplants.

These long-term follow-up results of early MAT cases appear less than promising. However, it should be taken into consideration that Milchowski et al. ${ }^{19)}$ used contralateral lateral menisci for medial meniscus transplantation in 11 of the 22 patients in the absence of established surgical techniques without recognition of the importance of joint congruity and graft size. Recent biomechanical studies have shown that placement of an inappropriately sized graft has a negative impact on the functional recovery of the meniscus. ${ }^{9,20,21)}$ Precise implantation of an appropriately sized allograft using a proper surgical technique is essential for good prognosis of $\mathrm{MAT}^{22-25)}$. In addition, lyophilized grafts were used in many of the early MATs and medial MATs were performed as an additional procedure in ACL reconstruction. Therefore, we think that the long-term efficacy of MAT should be evaluated based on studies that involve a high volume of cases and the use of currently preferred cryopreserved or deep frozen size-matched grafts.

The mid-term failure rate of cryopreserved graft transplantation was as high as $35 \%{ }^{26-28}$. In a study by Strollsteimer et al. ${ }^{26)}, 8(35 \%)$ out of 23 patients required a second operation due to meniscal symptoms at 13-69 months after MAT. In a 2- to 8-year followup study by Rath et al. ${ }^{27}$, transplant removal was necessitated in $8(36 \%)$ out of 22 cases with a cryopreserved graft at a mean of 31 months postoperatively. van Arkel and de Boer ${ }^{28)}$ analyzed the survival rate of cryopreserved meniscal allografts at a mean of 60 months of follow-up. When failure was defined as persistent pain and meniscal transplant tear, the cumulative survival rates of the lateral, medial, and bilateral allografts were $76 \%, 50 \%$, and $67 \%$, respectively. The failure rate was high in the ACL-deficient knee with ligament instability.

There are some recently reported long-term follow-up studies that demonstrate more favorable results. Hommen et al. ${ }^{29)}$ followed 20 cases for a mean of 141 months after cryopreserved graft transplantation and observed improvement in the Lysholm score and pain score in $90 \%$ of the patients. However, when failure was defined as $<65$ Lysholm score or no improvement in pain score, the failure rate was $35 \%$. On the objective evaluation results, radiographic joint space narrowing was observed in 10 out of 15 patients, moderate meniscus shrinkage was noted in all of the 7 patients who underwent MRI, 3 of which had grade III signal intensities. Thus, the 10-year survival rate of the allografts based on the clinical outcome and the objective evaluation results became $45 \%$ (9 in 20 patients). van der Wal et al. ${ }^{30)}$ assessed the 
results of 63 open meniscal transplantation procedure using a cryopreserved graft at a mean of 13.8 years postoperatively. They suggested that the procedure would be a good salvage option with an overall failure rate of $29 \%$ in the meniscectomized knee in spite of functional deterioration (Lysholm score, 61 points) at the last follow-up.

\section{Graft Survivorship and Concomitant Realignment Surgery}

Ligament instability and malalignment lead to abnormal load bearing on the affected compartment of the knee, which can be the cause of early failure of an $\mathrm{MAT}^{31,32)}$. van Arkel and de Boer ${ }^{32)}$ reported that isolated cryopreserved graft transplantation failed at a mean of 2 years postoperatively because the graft had become detached from the capsule in 3 cases with a poor Lysholm score out of 23 cases. In 5 of 12 cases that were available for secondlook arthroscopy, the graft was detached from the capsule due to revascularization failure caused by malalignment. Thereafter, the importance of malalignment correction is well documented in several studies. In a study by Cameron and Saha ${ }^{31)}$, although an advanced osteoarthritic change was present in all of the 67 cases (63 patients), the results were good to excellent in 29 (85.3\%) of 34 cases that underwent realignment osteotomy in combination with MAT. In a study by Verdonk et al. ${ }^{33)}$, when failure was defined as moderate occasional or persistent pain or poor function, $28 \%$ of the medial and $16 \%$ of the lateral MATs failed out of a total of 100 MATs at a mean of 7.2 years postoperatively. The cumulative survival rates at 10 years postoperatively were $74.2 \%$ for the medial allografts and $69.8 \%$ for the lateral allografts. In cases where medial MAT was performed in combination with a HTO, the survival rate was $83.3 \%$. However, their analysis was based on the clinical scoring and symptoms without any comparison with a control group where only HTO was performed. Considering that the 10-year cumulative survival rate of HTO in recent studies were as high as $79 \%{ }^{34)}$ and $92 \%{ }^{35)}$, it is difficult to determine whether the $83.3 \%$ of high success rate can be attributable to HTO or the synergistic effect of the two procedures in the MAT with HTO group. In addition, we think the condition of grafts itself should be evaluated in an objective manner, such as MRI scan or second-look arthroscopy. Verdonk et al. ${ }^{24)}$ assessed the clinical and radiological outcomes of 42 cases of MAT with a minimum follow-up of 10 years. The Hospital for Special Surgery score was higher in the MAT with HTO group than in the isolated MAT group. There was no progression of joint space narrowing on radiography in 13 (41\%) out of 32 cases. Fairbank changes were stable in 9 of the 32 cases. MRI showed no progression of cartilage degeneration in 6 (35\%) out of 17 cases, but an increased signal intensity of the meniscal allograft and graft extrusion were present in most of the patients at the last follow-up. The 10-year cumulative success rate was more than $80 \%$ including 7 cases (18\%) that underwent total knee arthroplasty (TKA). However, the study did not include an objective analysis on the graft condition and HTO.

\section{Preexisting Cartilage Degeneration of MAT Candidates}

Although MAT has been known to produce poor results in the knee with preexisting cartilage degeneration ${ }^{10,32,36)}$, some recent studies have shown the results of MAT combined with a cartilage procedure. Stone et al. ${ }^{37)}$ reported the results of 119 transplantations of meniscus allografts (94 deep frozen grafts, 24 cryopreserved grafts, and 1 irradiated graft) that were performed concurrently with cartilage repair in patients with severe articular cartilage damage. During a mean 5.8 years of follow-up, failure occurred in 25 cases (20.1\%) at a mean of 4.6 years postoperatively, and TKA was necessitated in 18 cases at a mean of 5.1 years postoperatively. The mean survival time for the whole series was 9.9 years and this is relatively good results considering that 53 cases $(46 \%)$ were more than 50 years of age and preoperative severe arthrosis was present in all cases. Harris et al. ${ }^{12)}$ conducted a systemic review of 6 studies on MAT combined with a cartilage repair procedure, such as autologous chondrocyte implantation $(n=73)$, osteochondral allograft transfer $(n=20)$, osteochondral autograft transfer $(n=17)$, and microfracture $(n=3)$. The outcomes of the combined procedures were comparable to those of either procedure performed alone in 4 of the 6 studies, whereas less favorable in the other 2 studies, and the overall failure rate was $12 \%$.

\section{Evaluation Methods for Meniscal Transplants}

In most studies, the outcomes of MAT have been evaluated using clinical parameters, such as the Lysholm score, International Knee Documentation Committee score, and visual analog scale. Therefore, the proven benefits of the procedure are pain relief and functional improvement. However, these clinical assessments do not accurately reflect the status of meniscal transplants. It is more worthwhile to thoroughly assess graft condition itself and joint preservation effect in objective evaluation studies with a high level of evidence. Arthroscopy is the most accurate objective 
evaluation method but is an invasive modality. Thus, MRI scans are more commonly used as a relatively reliable and noninvasive evaluation method. Noyes et al. ${ }^{36)}$ used MRI in 29 out of 40 cases at a mean of 35 months postoperatively, which showed more than grade 3 signal intensity in 3 cases, and knee joint arthrosis (normal/mild in 22 cases and moderate in 18 cases).

Recent short- or mid-term objective evaluation studies provide more favorable results than past studies in the literature. Ha et al. ${ }^{38)}$ performed second-look arthroscopy at a minimum of 2 years after medial MAT in 11 out of 22 cases, which revealed complete healing in more than $80 \%$ of the patients and cartilage degeneration in 4 cases (36.4\%). Kim et al. ${ }^{39)}$ confirmed with MRI or second-look arthroscopy that the allografts were graded as satisfactory in 20 cases (69.0\%), fair in 5 cases $(17.2 \%)$ at a minimum of 2 years after 29 isolated lateral MAT. Zhang et al. ${ }^{40)}$ observed failure in $2(11 \%)$, alteration of characteristics in $10(56 \%)$, and normal characteristics in $6(33 \%)$ out of 18 cases on second-look arthroscopy performed at a mean of 2 years after MAT. In a study by Marcacci et al. ${ }^{41)}$, pain relief and functional improvement were obtained in $94 \%$ of 32 cases at a mean of 40 months after MAT and MRI showed improved cartilage condition on the femoral side and tibial side at a mean of 36 months postoperatively. Kim et al. ${ }^{42)}$ reported objective evaluation results of MAT using bone fixation. In their series, MRI or second-look arthroscopy was performed in 110 (95.7\%) out of 115 cases at more than 2 years postoperatively and the results were graded as satisfactory in $90(81.8 \%)$, fair in $8(7.3 \%)$, and poor in 12 cases (10.9\%).

\section{Meniscal Transplants Not Better Than Normal But Maybe Better Than Absent}

A certain level of degeneration or tear is commonly observed in the meniscal transplant ${ }^{8}$. Therefore, the orthopedic surgeons should keep in mind that transplanted menisci cannot restore perfectly the normal meniscal function but just improve functions with a possible chondroprotective effect in the meniscectomized $\mathrm{knee}^{433}$. In addition, patients should be educated beforehand about the postoperative knee joint condition that cannot be comparable to normal knee joint due to the preexisting articular cartilage damage and advised to engage in light sports activities. The original meniscus cannot be supplanted by MAT, and therefore total or sub-total meniscectomy should not be decided easily in young patients if the status of a torn meniscus allows suture repair.

On the other hand, MAT can result in poor outcomes after the patients present with severe painful joint that are typical indications of MAT $^{10,32,36)}$, because advanced articular cartilage damage may be present frequently in such patients ${ }^{12,36,37}$. It is our understanding that even in the presence of mild symptoms, if significant articular cartilage damage is present or progression of the damage is noted on high-resolution MRI, early decision for MAT should be considered for the good prognosis.

\section{Conclusions}

MAT is a useful treatment method for functional improvement and symptomatic relief in the symptomatic meniscectomized knee. It has been established in mid- and long-term studies that MAT is effective for reduction in pain and swelling and improvement of knee function. In addition, some animal studies have shown that the technique provides a chondroprotective effect after meniscectomy, although the protection is not complete compared to the normal meniscus ${ }^{15,44}$. However, there are not sufficient comparison studies with high level evidence, such as randomized controlled studies, for determining its efficacy in human subjects. In addition, more long-term followup studies should be conducted to assess the effect of preventive application of MAT.

\section{Conflict of Interest}

No potential conflict of interest relevant to this article was reported.

\section{References}

1. Jeong HJ, Lee SH, Ko CS. Meniscectomy. Knee Surg Relat Res. 2012;24:129-36.

2. Cho JH. Modified pull-out suture in posterior root tear of the medial meniscus: using a posteromedial portal. Knee Surg Relat Res. 2012;24:124-7.

3. Fairbank TJ. Knee joint changes after meniscectomy. J Bone Joint Surg Br. 1948;30:664-70.

4. Radin EL, de Lamotte F, Maquet P. Role of the menisci in the distribution of stress in the knee. Clin Orthop Relat Res. 1984;(185):290-4.

5. Levy IM, Torzilli PA, Gould JD, Warren RF. The effect of lateral meniscectomy on motion of the knee. J Bone Joint Surg Am. 1989;71:401-6.

6. Levy IM, Torzilli PA, Warren RF. The effect of medial meniscectomy on anterior-posterior motion of the knee. J 
Bone Joint Surg Am. 1982;64:883-8.

7. Bargar WL, Moreland JR, Markolf KL, Shoemaker SC, Amstutz HC, Grant TT. In vivo stability testing of postmeniscectomy knees. Clin Orthop Relat Res. 1980;(150):24752.

8. Noyes FR, Barber-Westin SD. Repair of complex and avascular meniscal tears and meniscal transplantation. J Bone Joint Surg Am. 2010;92:1012-29.

9. Matava MJ. Meniscal allograft transplantation: a systematic review. Clin Orthop Relat Res. 2007;455:142-57.

10. Rodeo SA. Meniscal allografts: where do we stand? Am J Sports Med. 2001;29:246-61.

11. Lee SR, Kim JG, Nam SW. The tips and pitfalls of meniscus allograft transplantation. Knee Surg Relat Res. 2012;24:13745.

12. Harris JD, Cavo M, Brophy R, Siston R, Flanigan D. Biological knee reconstruction: a systematic review of combined meniscal allograft transplantation and cartilage repair or restoration. Arthroscopy. 2011;27:409-18.

13. Hergan D, Thut D, Sherman O, Day MS. Meniscal allograft transplantation. Arthroscopy. 2011;27:101-12.

14. von Lewinski G, Pressel T, Hurschler C, Witte F. The influence of intraoperative pretensioning on the chondroprotective effect of meniscal transplants. Am J Sports Med. 2006;34:397-406.

15. Kelly BT, Potter HG, Deng XH, Pearle AD, Turner AS, Warren RF, Rodeo SA. Meniscal allograft transplantation in the sheep knee: evaluation of chondroprotective effects. Am J Sports Med. 2006;34:1464-77.

16. Wirth CJ, Peters G, Milachowski KA, Weismeier KG, Kohn D. Long-term results of meniscal allograft transplantation. Am J Sports Med. 2002;30:174-81.

17. von Lewinski G, Milachowski KA, Weismeier K, Kohn D, Wirth CJ. Twenty-year results of combined meniscal allograft transplantation, anterior cruciate ligament reconstruction and advancement of the medial collateral ligament. Knee Surg Sports Traumatol Arthrosc. 2007;15:1072-82.

18. Binnet MS, Akan B, Kaya A. Lyophilised medial meniscus transplantations in ACL-deficient knees: a 19-year followup. Knee Surg Sports Traumatol Arthrosc. 2012;20:109-13.

19. Milachowski KA, Weismeier K, Wirth CJ. Homologous meniscus transplantation: experimental and clinical results. Int Orthop. 1989;13:1-11.

20. Dienst M, Greis PE, Ellis BJ, Bachus KN, Burks RT. Effect of lateral meniscal allograft sizing on contact mechanics of the lateral tibial plateau: an experimental study in human cadaveric knee joints. Am J Sports Med. 2007;35:34-42.

21. Wilcox TR, Goble EM, Doucette SA. Goble technique of meniscus transplantation. Am J Knee Surg. 1996;9:37-42.

22. Canham W, Stanish W. A study of the biological behavior of the meniscus as a transplant in the medial compartment of a dog's knee. Am J Sports Med. 1986;14:376-9.

23. Rue JP, Yanke AB, Busam ML, McNickle AG, Cole BJ. Prospective evaluation of concurrent meniscus transplantation and articular cartilage repair: minimum 2-year followup. Am J Sports Med. 2008;36:1770-8.

24. Verdonk PC, Verstraete KL, Almqvist KF, De Cuyper K, Veys EM, Verbruggen G, Verdonk R. Meniscal allograft transplantation: long-term clinical results with radiological and magnetic resonance imaging correlations. Knee Surg Sports Traumatol Arthrosc. 2006;14:694-706.

25. Prodromos CC, Joyce BT, Keller BL, Murphy BJ, Shi K. Magnetic resonance imaging measurement of the contralateral normal meniscus is a more accurate method of determining meniscal allograft size than radiographic measurement of the recipient tibial plateau. Arthroscopy. 2007;23:1174-79.e1.

26. Stollsteimer GT, Shelton WR, Dukes A, Bomboy AL. Meniscal allograft transplantation: a 1- to 5-year follow-up of 22 patients. Arthroscopy. 2000;16:343-7.

27. Rath E, Richmond JC, Yassir W, Albright JD, Gundogan F. Meniscal allograft transplantation: two- to eight-year results. Am J Sports Med. 2001;29:410-4.

28. van Arkel ER, de Boer HH. Survival analysis of human meniscal transplantations. J Bone Joint Surg Br. 2002;84:22731.

29. Hommen JP, Applegate GR, Del Pizzo W. Meniscus allograft transplantation: ten-year results of cryopreserved allografts. Arthroscopy. 2007;23:388-93.

30. van der Wal RJ, Thomassen BJ, van Arkel ER. Long-term clinical outcome of open meniscal allograft transplantation. Am J Sports Med. 2009;37:2134-9.

31. Cameron JC, Saha S. Meniscal allograft transplantation for unicompartmental arthritis of the knee. Clin Orthop Relat Res. 1997;(337):164-71.

32. van Arkel ER, de Boer HH. Human meniscal transplantation: preliminary results at 2 to 5 -year follow-up. J Bone Joint Surg Br. 1995;77:589-95.

33. Verdonk PC, Demurie A, Almqvist KF, Veys EM, Verbruggen G, Verdonk R. Transplantation of viable meniscal allograft: survivorship analysis and clinical outcome of one hundred cases. J Bone Joint Surg Am. 2005;87:715-24.

34. Hui C, Salmon LJ, Kok A, Williams HA, Hockers N, van 
der Tempel WM, Chana R, Pinczewski LA. Long-term survival of high tibial osteotomy for medial compartment osteoarthritis of the knee. Am J Sports Med. 2011;39:64-70.

35. Schallberger A, Jacobi M, Wahl P, Maestretti G, Jakob RP. High tibial valgus osteotomy in unicompartmental medial osteoarthritis of the knee: a retrospective follow-up study over 13-21 years. Knee Surg Sports Traumatol Arthrosc. 2011;19:122-7.

36. Noyes FR, Barber-Westin SD, Rankin M. Meniscal transplantation in symptomatic patients less than fifty years old. J Bone Joint Surg Am. 2004;86:1392-404.

37. Stone KR, Adelson WS, Pelsis JR, Walgenbach AW, Turek TJ. Long-term survival of concurrent meniscus allograft transplantation and repair of the articular cartilage: a prospective two- to 12-year follow-up report. J Bone Joint Surg Br. 2010;92:941-8.

38. Ha JK, Sung JH, Shim JC, Seo JG, Kim JG. Medial meniscus allograft transplantation using a modified bone plug technique: clinical, radiologic, and arthroscopic results. Arthroscopy. 2011;27:944-50.

39. Kim CW, Kim JM, Lee SH, Kim JH, Huang J, Kim KA, Bin SI. Results of isolated lateral meniscus allograft transplantation: focus on objective evaluations with magnetic resonance imaging. Am J Sports Med. 2011;39:1960-7.

40. Zhang H, Liu X, Wei Y, Hong L, Geng XS, Wang XS, Zhang J, Cheng KB, Feng H. Meniscal allograft transplantation in isolated and combined surgery. Knee Surg Sports Traumatol Arthrosc. 2012;20:281-9.

41. Marcacci M, Zaffagnini S, Marcheggiani Muccioli GM, Grassi A, Bonanzinga T, Nitri M, Bondi A, Molinari M, Rimondi E. Meniscal allograft transplantation without bone plugs: a 3-year minimum follow-up study. Am J Sports Med. 2012;40:395-403.

42. Kim JM, Lee BS, Kim KH, Kim KA, Bin SI. Results of meniscus allograft transplantation using bone fixation: 110 cases with objective evaluation. Am J Sports Med. 2012;40:1027-34.

43. Kim JG, Lee YS, Bae TS, Ha JK, Lee DH, Kim YJ, Ra HJ. Tibiofemoral contact mechanics following posterior root of medial meniscus tear, repair, meniscectomy, and allograft transplantation. Knee Surg Sports Traumatol Arthrosc. 2012 Sep 7 [Epub]. http://10.1007/s00167-012-2182-4.

44. Szomor ZL, Martin TE, Bonar F, Murrell GA. The protective effects of meniscal transplantation on cartilage: an experimental study in sheep. J Bone Joint Surg Am. 2000;82:80-8. 